\title{
A simple model for studying multilayer adsorption of noninteracting polyatomic species on homogeneous and heterogeneous surfaces
}

\author{
F. O. Sánchez-Varretti, ${ }^{1}$ G. D. García, ${ }^{1}$ A. J. Ramirez-Pastor, ${ }^{2, a)}$ and F. Romá ${ }^{2,3, a)}$ \\ ${ }^{1}$ Universidad Tecnológica Nacional, Regional San Rafael, Gral. Urquiza 314, 5600 San Rafael, \\ Mendoza, Argentina \\ ${ }^{2}$ Departamento de Física, Instituto de Física Aplicada, Universidad Nacional de San Luis-CONICET, \\ Chacabuco 917, D5700BWS San Luis, Argentina \\ ${ }^{3}$ Centro Atómico Bariloche, San Carlos de Bariloche, Río Negro R8402AGP, Argentina
}

(Received 21 December 2008; accepted 14 April 2009; published online 21 May 2009)

\begin{abstract}
In this work we study a simple model of multilayer adsorption of noninteracting polyatomic species on homogeneous and heterogeneous surfaces. A new approximate analytic isotherm is obtained and validated by comparing with Monte Carlo simulation in one- and two-dimensional lattices. Then, we use the well-known Brunauer-Emmet-Teller (BET) approach to analyze these isotherms and to estimate the monolayer volume, $v_{m}$. In this way, we confirm previous observations that the value of the $v_{m}$ obtained by the BET equation depends strongly on adsorbate size and surface heterogeneity. In all cases, we find that the use of the BET equation leads to an underestimate of the true monolayer capacity. Nevertheless, a compensation effect is found for the adsorption on a patchwise bivariate surface, but this is not enough to eliminate the decrease of $v_{m}$ caused by the molecular size. In addition, we consider also the possibility to use the model to study the adsorption on nanotube bundles. (C) 2009 American Institute of Physics. [DOI: 10.1063/1.3139301]
\end{abstract}

\section{INTRODUCTION}

The theoretical description of multilayer adsorption is a long-standing important problem in surface science that does not have a general solution. ${ }^{1,2}$ Mainly this is due to the fact that the structure of the different layers differs from that in contact with the solid surface (first layer). At high coverage (multilayer region), it is expected that the adsorption process is well described by the slab theory of Frenkel, ${ }^{3}$ Halsey, ${ }^{4}$ and Hill. ${ }^{5}$ In this approach it is assumed that the higher layers retain the structure of the bulk liquid and only its free energy changes gradually as one goes away from the solid surface. On the other hand, at low coverage, it is more appropriate to use the Brunauer-Emmet-Teller (BET) isotherm, ${ }^{6}$ where the crystal-like structure of the surface is considered. In the BET theory it is assumed that the molecules are localized in sites and that the adsorption in the first layer is different from the remaining ones.

The BET equation is one of the most widely used tools to analyze experimental isotherms and to determine the monolayer volume (or monolayer capacity) of the a solid surface. This approach neglects such things as the polyatomic character of the adsorbate, the interaction between the admolecules, and the surface heterogeneities. Thus, with the purpose of including a more complex situation, numerous generalizations of the BET theory have been proposed. ${ }^{2,7-9}$ These leading models, along with more recent contributions, ${ }^{10-12}$ played a central role in the characterization of solid surfaces by means of gas adsorption. Nevertheless, the simplicity of the original BET isotherm made it very

\footnotetext{
${ }^{a)}$ Author to whom correspondence should be addressed. Electronic mail: antorami@unsl.edu.ar.
}

popular for practical purposes. In fact, by fitting an experimental isotherm with the BET equation, in many cases, it is possible to predict the monolayer volume with an error not bigger than the $20 \%{ }^{1}$ This surprising result is attributed to compensation effects arising as consequence of having carried out many approximations. ${ }^{1}$

By means of numerical experiments, Walker and Zettlemoyer $^{13}$ have shown that the conventional BET theory predicts a monolayer volume smaller than the real value when heterogeneous adsorption isotherms are analyzed. A similar result was obtained by Cortés and Araya ${ }^{14}$ when considering a Gaussian distribution of adsorption energies. The authors showed that the estimated values of the monolayer volume from the BET equation diminishes with increasing degree of surface heterogeneity (the width of the distribution of adsorption energies).

More recently, Nikitas ${ }^{15}$ arrived to similar conclusions by considering both surface heterogeneity and polyatomic character of the adsorbate. In Ref. 15, by using an extension of the Flory-Huggins polymer solution theory, ${ }^{7}$ the multilayer adsorption of polyatomic species was studied over a random heterogeneous surface. The author concludes that one can obtain an underestimation of the true monolayer capacity of the order of $25 \%$, when the adsorbate occupies more than one lattice site. This underestimation is bigger if a heterogeneous surface is considered.

Although these studies show that in many situations the true monolayer volume is underestimated, it is necessary to stress that this is not a general rule because random heterogeneous surfaces were always considered and the polyatomic character of the adsorbate was only taken into account in Ref. 15. As we show in this work, surface topography (the space distribution of the adsorption energies over the solid 
surface) is very important, since it also affects the predictions of the BET equation. However, although for the adsorption on a patchwise bivariate surface a compensation effect is found, we determine that this is not enough to eliminate the decrease in the estimated value of the monolayer volume caused by the molecular size. Only a total compensation effect has been observed in multilayer adsorption on homogeneous surface, when polyatomic adsorbates and attractive lateral interactions were considered. ${ }^{16}$

In this work, we study how the monolayer volume predicted by BET equation differs from its real value when considering both the adsorbate size and the surface topography. With this purpose in mind, first we define a simple model of multilayer adsorption of noninteracting polyatomic species on one-dimensional (1D) and two-dimensional (2D) homogeneous and heterogeneous bivariate surfaces. This model is a generalization of the BET theory. In each case, analytic isotherms are built and validated by comparing with Monte Carlo (MC) simulation. Then, by analyzing these isotherms with the conventional BET theory, we estimate the monolayer volume for different conditions of adsorption (different topographies, adsorbate size, and surface dimensionality). In addition, we analyze the qualitative behavior of the 1D model for binding energy and temperature characteristics of the adsorption of ethane on samples of single-walled nanotubes.

The paper is structured as follows. In Sec. II we present the model of the multilayer adsorption of noninteracting polyatomic species on homogeneous and heterogeneous bivariate surfaces. Next, in Secs. III and IV, exact and approximate isotherms are obtained and compared with MC simulation. The dependence of the monolayer volume on the surface topography (patchwise) and the adsorbate size is presented in Sec. V. In Sec. VI, it is considered also the possibility to use the 1D model to study the adsorption on nanotube bundles. Finally the conclusions are drawn in Sec. VII.

\section{MODEL}

A simple lattice-gas model to describe the multilayer adsorption of noninteracting polyatomic molecules on homogeneous surface has been recently proposed. ${ }^{17,18}$ The surface is modeled by a regular lattice of $M$ sites with periodic boundary conditions, where all the sites have the same adsorption energy $\varepsilon$ and the adsorbate is represented by $k$-mers (linear particles that have $k$ identical units). A $k$-mer adsorbed on the surface occupies $k$ sites of the lattice with an energy $k \varepsilon$ and can arrange in many configurations. This property is called adsorption with multisite occupancy. On the other hand, for higher layers, the adsorption of a $k$-mer is exactly onto an already absorbed one, with an adsorption energy of $k U$. Thus, the monolayer structure reproduces in the remaining layers. Finally, following the spirit of the BET theory, no lateral interactions are considered and only interactions among the layers are introduced. Figure 1 in Ref. 17 shows a snapshot representing this lattice-gas model.

In our model, the mechanism used to describe the adsorption in the multilayer regime mimics the phenomenon called pseudomorphism. This was observed, by using low- energy electron diffraction technique, ${ }^{19-21}$ in the case of adsorption of straight chain saturated hydrocarbon molecules on metallic surfaces. However, recent synchrotron x-ray scattering measurements show a different and more complex growth process in these systems. ${ }^{22}$ Nevertheless, for simplicity, here we study a variant of the model that maintains the same mechanism to describe the adsorption in the higher layers. Because we analyze isotherms in the low coverage regime, we expect that this choice will not affect our results.

We modify the system in Refs. 17 and 18 to consider the adsorption on a heterogeneous substrate: now the adsorption energy $\varepsilon_{i}$ depends on each site $i$ of the surface. Then, the Hamiltonian of the system is

$$
H=\left(N-N_{m}\right) k U+\sum_{i=1}^{M} \sigma_{i} \varepsilon_{i},
$$

where $N$ is the total number of $k$-mers, $\sigma_{i}$ the occupation variable that can take the values 0 if the site $i$ is empty or 1 if the site $i$ is occupied, and $N_{m}$ is the number of $k$-mers on the surface (monolayer),

$$
N_{m}=\frac{1}{k} \sum_{i=1}^{M} \sigma_{i}
$$

The Hamiltonian in Eq. (1) can be rewritten as

$$
H=N k U+\sum_{i=1}^{M} \sigma_{i}\left(\varepsilon_{i}-U\right) .
$$

In the following sections, we will look for analytic solutions of the model in 1D and 2D homogeneous and heterogeneous surfaces.

\section{MULTILAYER ADSORPTION ON HOMOGENEOUS SURFACES}

In the previous model, a simple analytic expression of the multilayer adsorption isotherm can be obtained in few particular cases. If the surface is homogeneous $\left(\varepsilon_{i}=\varepsilon\right.$ for all i) and $k=1$, it is easy to demonstrate ${ }^{1,2,17}$ that

$$
\theta=\frac{1}{\left(1-p / p_{0}\right)} \frac{c p / p_{0}}{\left[1+(c-1) p / p_{0}\right]} .
$$

Here, $\theta=k N / M$ is the total coverage, $p$ is the pressure, $p_{0}$ is the saturation pressure of the bulk liquid, and $c$ is a constant defined as

$$
c=\exp [-\beta k(\varepsilon-U)],
$$

where $\beta=1 / k_{B} T$ is the inverse temperature ( $k_{B}$ being the Boltzmann constant and $T$ the absolute temperature). Equation (4) is the well-known BET isotherm ${ }^{6}$ and can be applied to systems in any dimension.

In the case of $k=2$, it is only possible to obtain an exact solution in 1D (Ref. 17),

$$
\theta=\frac{1}{\left(1-p / p_{0}\right)}\left\{1-\left[\frac{1-p / p_{0}}{1+(4 c-1) p / p_{0}}\right]^{1 / 2}\right\} .
$$

Equation (6) is the exact dimer isotherm for the multilayer adsorption on 1D homogeneous surfaces. As it has been pre- 
viously shown, ${ }^{17}$ the values of the monolayer volume predicted by Eqs. (4) and (6) are different: if a dimer isotherm is analyzed, the value of the monolayer capacity arising from using BET characterization is smaller than the real one.

In general, the multilayer isotherm corresponding to the model of Eq. (3) cannot be expressed by one equation only and it is necessary to give two functions. ${ }^{15,18}$ Let us assume that an analytical expression of the monolayer adsorption isotherm is known, $\exp (\mu \beta)=\lambda, \mu$ being the chemical potential and $\lambda$ (the fugacity) a function of the monolayer coverage $\theta_{m}=k N_{m} / M$. Then, the following equations can be deduced: ${ }^{18}$

$$
\frac{p}{p_{0}}=\frac{\lambda\left(\theta_{m}\right)}{c+\lambda\left(\theta_{m}\right)}
$$

and

$$
\theta=\frac{\theta_{m}}{\left(1+p / p_{0}\right)}
$$

Equations (7) and (8) constitute the multilayer adsorption isotherm. By using the surface coverage $\left(0 \leq \theta_{m} \leq 1\right)$ as a parameter, we can calculate the relative pressure from Eq. (7). Then, the values of $\theta_{m}$ and $p / p_{0}$ are introduced in Eq. (8) and the total coverage is obtained.

Following the previous scheme, it is possible to obtain the exact multilayer isotherm for $k$-mers in 1D homogeneous surfaces. ${ }^{18}$ We start from the exact monolayer isotherm ${ }^{23,24}$

$$
\lambda=\frac{\theta_{m}\left[1-\frac{(k-1)}{k} \theta_{m}\right]^{k-1}}{k\left(1-\theta_{m}\right)^{k}} .
$$

Then, Eq. (7) can be written as

$$
\frac{p}{p_{0}}=\frac{\theta_{m}\left[1-\frac{(k-1)}{k} \theta_{m}\right]^{k-1}}{k c\left(1-\theta_{m}\right)^{k}+\theta_{m}\left[1-\frac{(k-1)}{k} \theta_{m}\right]^{k-1}} .
$$

Equations (8) and (10) represent the exact solution of the 1D model. In particular, for $k=1$ and $k=2$, it is possible to solve

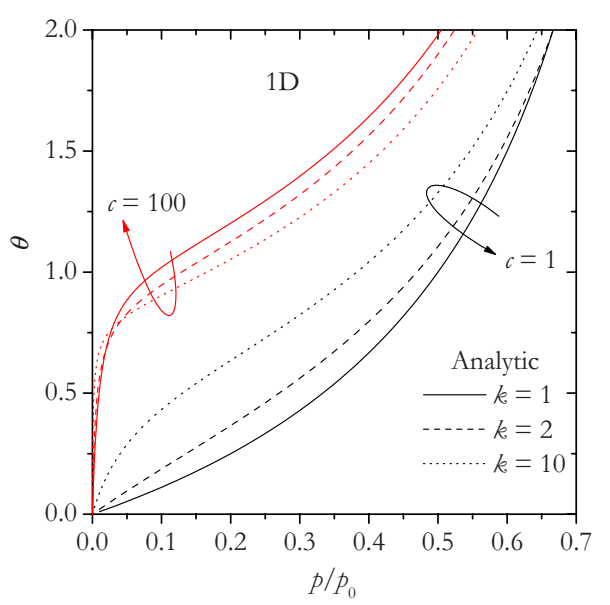

FIG. 1. (Color online) Exact 1D isotherms for $k=1,2$, and 10, and two values of $c$ as indicated.

these equations to obtain single expressions of the multilayer isotherms, Eqs. (4) and (6), respectively. Figure 1 shows the exact 1D isotherms for $k=1,2$, and 10, and two values of $c$.

Also, the previous scheme can be used to obtain an accurate approximation for multilayer adsorption on 2D substrates accounting multisite occupancy. In this case, we use the semiempirical (SE) monolayer adsorption isotherm ${ }^{25,26}$

$$
\lambda=\frac{\theta_{m}\left[1-\frac{(k-1)}{k} \theta_{m}\right]^{(k-1) \theta_{m}}\left[1-\frac{2(k-1)}{\gamma k} \theta_{m}\right]^{(k-1)\left(1-\theta_{m}\right)}}{\zeta k\left(1-\theta_{m}\right)^{k}},
$$

where $\gamma$ is the connectivity of the lattice and $\zeta$ represents the number of available configurations (per lattice site) for a linear $k$-mer at zero coverage. Thus, $\zeta=1$ for $k=1$ and $\zeta=\gamma / 2$ for $k \geq 2$. It has been shown that Eq. (11) is a very good approximation for representing multisite-occupancy adsorption in the monolayer regime. ${ }^{25,26}$ Then, by using Eqs. (7) and (11), we obtain

$$
\frac{p}{p_{0}}=\frac{\theta_{m}\left[1-\frac{(k-1)}{k} \theta_{m}\right]^{(k-1) \theta_{m}}\left[1-\frac{2(k-1)}{\gamma k} \theta_{m}\right]^{(k-1)\left(1-\theta_{m}\right)}}{\zeta k c\left(1-\theta_{m}\right)^{k}+\theta_{m}\left[1-\frac{(k-1)}{k} \theta_{m}\right]^{(k-1) \theta_{m}}\left[1-\frac{2(k-1)}{\gamma k} \theta_{m}\right]^{(k-1)\left(1-\theta_{m}\right)}} .
$$

Note that, for $\gamma=2$, the SE isotherm is identical to Eq. (9). Therefore, Eqs. (8) and (12) represent the general solution of the problem of multilayer adsorption in homogeneous surfaces with multisite occupancy. For $\gamma=2$ (1D), this isotherm is exact, but it is approximate for $\gamma>2$. In addition, in any dimension, the exact isotherm for $k=1$ (BET equation) can also be obtained from Eqs. (8) and (12).

In order to test the 2D approximation, we compared the analytic multilayer isotherm with results of MC simulation. The algorithm used is described in Ref. 18. Here, the equi- 

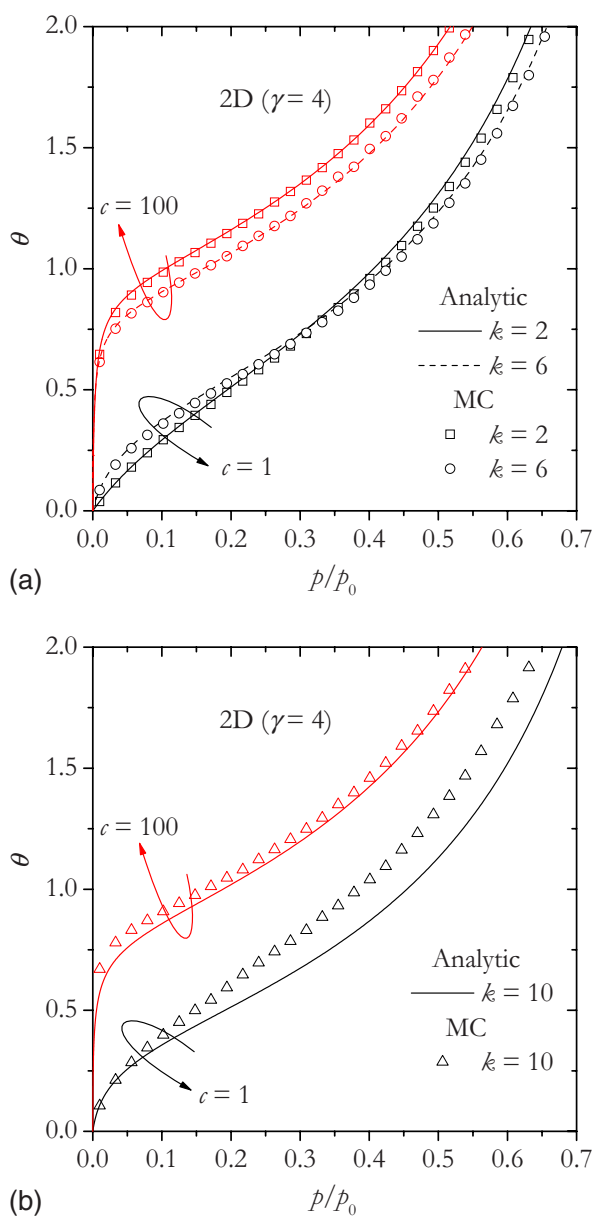

FIG. 2. (Color online) Comparison between analytic and simulated adsorption isotherms for 2D lattices and two different values of $c$ as indicated. (a) $k=2$ and 6, (b) $k=10$. In all cases, we used $t=10^{5}$ MCSs.

librium state is reproduced after discarding a number $t$ of MC steps (MCSs). Then, the mean value of the total coverage is obtained as

$$
\theta=\frac{k\langle N\rangle}{M},
$$

where the average $\langle N\rangle$ is calculated over another $t$ successive MCSs (the total number of MCSs is $2 t$ ). The computational simulations were developed for a square lattice $(\gamma=4)$ of linear size $L(M=L \times L)$. For each value of $k$, we choose $L$ $=20 k$. For these lattice sizes we verified that finite-size effects are negligible.

Figure 2(a) shows a comparison between the analytic isotherm [given by Eqs. (8) and (12)] and the MC results, for $k=2$ and 6 and two values of $c$. As we can see, the agreement is very good for the parameters used in the figure. On the other hand, the accuracy of the analytic isotherm diminishes as $k$ increases. Figure 2(b) shows this effect for $k=10$. Also, in this figure, we can appreciate that the difference between the analytic and the numerical isotherms diminishes as $c$ is increased.

\section{MULTILAYER ADSORPTION ON HETEROGENEOUS SURFACES}

In the previous section, we obtained the multilayer isotherm from the monolayer isotherm. It is possible demonstrate that, in general, the formalism allows to establish this connection only if (1) pseudomorphism is present and (2) no lateral interactions between the molecules in the multilayer regime are considered. In fact, Eqs. (7) and (8) still hold if the particles in the monolayer interact among them and with the solid surface. Then, we could use this formalism to calculate the multilayer adsorption isotherm for noninteracting $k$-mers and a given surface heterogeneity. For this purpose, an appropriate monolayer isotherm could be approximated by a weighted sum of the monolayer homogeneous isotherms (see below). This strategy leads to a complex solution that is not useful for practical purposes. To build a simpler function (easier to analyze), we have chosen to approximate the multilayer heterogeneous isotherm by a weighted sum of multilayer homogeneous isotherms. Then, Eqs. (7) and (8) are used only to obtain the appropriate multilayer homogeneous isotherms.

We start here from the integral representation of the adsorption multilayer isotherm, ${ }^{2}$

$$
\theta=\int \chi(\varepsilon) \theta_{\mathrm{loc}}(\varepsilon) d \varepsilon
$$

where $\theta_{\mathrm{loc}}(\varepsilon)$ represents the local adsorption multilayer isotherm corresponding to an adsorptive site of energy $\varepsilon$ and $\chi(\varepsilon)$ is the adsorptive energy distribution, which characterizes the surface heterogeneity (as before, the total and the local coverage depend on $p$ and $T$ ). It should be noted that Eq. (14) is strictly and generally valid only for noninteracting monomers $(k=1)$, which is a quite unrealistic case. If adsorbed particles occupy more than one site or interact with each other, then the local coverage at a point with a given adsorptive energy depends on the local coverage on neighboring points with different adsorptive energies and, in general, Eq. (14) should be replaced by a much more complex expression. $^{27,28}$

Nevertheless, in some situations Eq. (14) represents a good approximation of the adsorption isotherm (see below). For a lattice-gas model of $k$-mers, we can generalize this equation as

$$
\theta=\sum_{s} \theta_{\mathrm{loc}}\left(E_{s}\right) .
$$

In the last equation, the sum extends over all possible configurations of a single $k$-mer in an empty lattice and $E_{s}$ is the adsorption energy of each one of them. Note that the values of $E_{s}$ depend, among other things, on the energy distribution $\chi(\varepsilon)$, the surface topography, and the number $k$.

In the following, we will study the multilayer adsorption on 1D and 2D heterogeneous surfaces. As local isotherm, we will use Eqs. (8) and (12). Then, we will compare the multilayer adsorption isotherm obtained by using Eq. (15) and the calculated with MC simulation. 

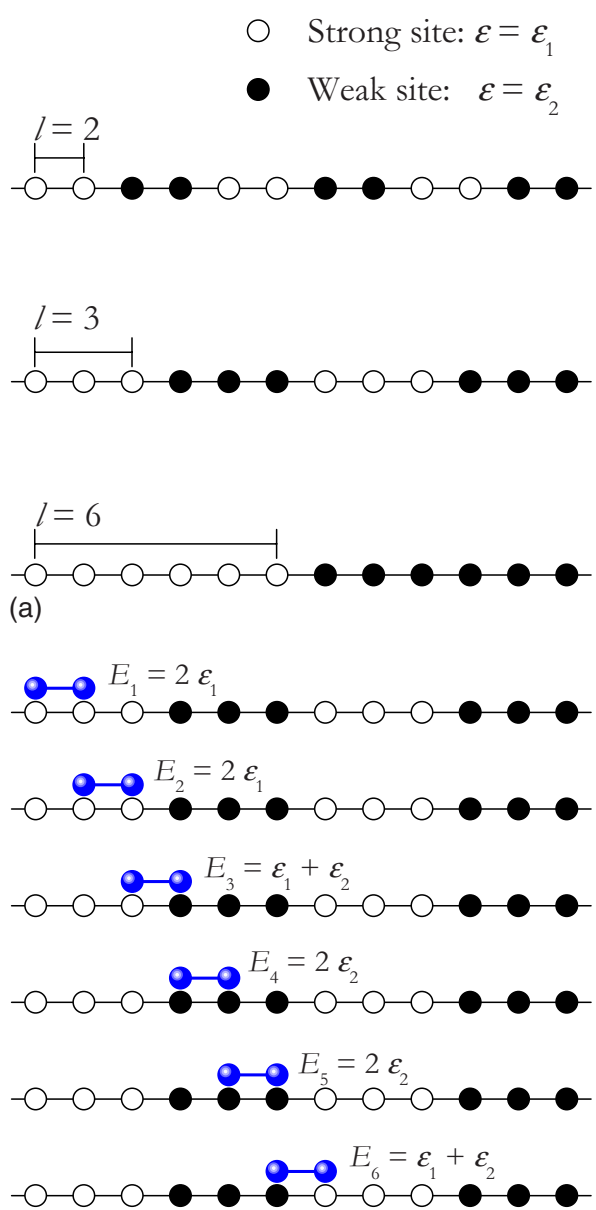

(b)

FIG. 3. (Color online) Cartoon representing the 1D lattice-gas model for a patchwise surface topography. (a) Strong and weak sites arrange in homotattic patches of length $l=2,3$, and 6 . (b) The six different configurations (and their adsorption energies) of a dimer on a surface with $l=3$.

\section{A. Adsorption on 1D heterogeneous surfaces}

The heterogeneous surface is modeled by two kinds of sites (bivariate surface): strong sites with adsorption energy $\varepsilon_{1}$ and weak sites with adsorption energy $\varepsilon_{2}\left(\varepsilon_{1}<\varepsilon_{2}\right)$. In 1D, these sites are spatially distributed in homotattic patches of length $l(l=1,2,3, \cdots)$. As an example, Fig. 3(a) shows three lattices with $l=2,3$, and 6 .

The number of possible configurations of a single $k$-mer in an empty lattice is $M$. However, due to periodicity, Eq. (15) has only $2 l$ terms (with many of them having the same adsorption energy). As an example, the six different configurations (and their adsorption energies) of a dimer on a patchwise surface with $l=3$ are shown in Fig. 3(b). Then, the multilayer isotherm is approximated as

$$
\theta=\frac{1}{2 l} \sum_{i=1}^{2 l} \theta_{\mathrm{loc}}\left(c_{i}\right) .
$$

Each term corresponds to an effective value of $c$ given by

$$
c_{i}=\exp \left[-\beta\left(E_{i}-k U\right)\right],
$$

where $E_{i}$ is the adsorption energy. This value of $c_{i}$ can also be expressed as function of $c_{1}$ and $c_{2}$, the values of $c$ for homogeneous surfaces given by Eq. (5) and whose adsorp- tion energies are $\varepsilon_{1}$ and $\varepsilon_{2}$, respectively. If the $i$ th term in Eq. (16) corresponds to a $k$-mer with $k_{1}$ units located over strong sites and $k_{2}$ units located over weak sites, then the adsorption energy is $E_{i}=k_{1} \varepsilon_{1}+k_{2} \varepsilon_{2}$, and

$$
c_{i}=\left(c_{1}^{k_{1}} c_{2}^{k_{2}}\right)^{1 / k} \text {. }
$$

As mentioned previously, we use Eqs. (8) and (12) with $\gamma=2$ as local isotherm. Note that Eq. (16) is a sum of local isotherms with different values of $c$, but at the same relative pressure. Then, in most of the cases it is necessary to be careful; although, for each local isotherm the surface coverage should be used as a parameter, it is not possible to use this as common parameter. In fact, Eq. (7) shows that for a fixed value of $p / p_{0}$, the surface coverage depends on $c$, $\theta_{m}\left(c_{i}\right)$.

Now, we analyze two simple cases. On one hand, Eq. (16) is exact for $k=1$ and can be obtained as the semisum of two BET isotherms,

$$
\begin{aligned}
\theta= & \frac{p / p_{0}}{2\left(1-p / p_{0}\right)}\left\{\frac{c_{1}}{\left[1+\left(c_{1}-1\right) p / p_{0}\right]}\right. \\
& \left.+\frac{c_{2}}{\left[1+\left(c_{2}-1\right) p / p_{0}\right]}\right\} .
\end{aligned}
$$

In this case, Eq. (19) does not depend on $l$ and, consequently, the multilayer adsorption isotherm is the same for all topography. On the other hand, Eq. (16) has three different terms for $k=2$, being each one of them a dimer isotherm Eq. (6) with a particular value of $c$. Thus, for $k=2$ the multilayer adsorption isotherm is

$$
\begin{aligned}
\theta= & \left(\frac{l-1}{2 l}\right) \frac{1}{\left(1-p / p_{0}\right)}\left\{1-\left[\frac{1-p / p_{0}}{1+\left(4 c_{1}-1\right) p / p_{0}}\right]^{1 / 2}\right\} \\
& +\left(\frac{1}{l}\right) \frac{1}{\left(1-p / p_{0}\right)}\left\{1-\left[\frac{1-p / p_{0}}{1+\left(4 \sqrt{c_{1} c_{2}}-1\right) p / p_{0}}\right]^{1 / 2}\right\} \\
& +\left(\frac{l-1}{2 l}\right) \frac{1}{\left(1-p / p_{0}\right)}\left\{1-\left[\frac{1-p / p_{0}}{1+\left(4 c_{2}-1\right) p / p_{0}}\right]^{1 / 2}\right\} .
\end{aligned}
$$

The first [third] term in the RHS of Eq. (20) represents the adsorption within a strong [weak] patch, on a pair of sites $(1,1)[(2,2)]$, with $c_{1}\left[c_{2}\right]$. There are $(l-1)$ configurations of this for each patch. The remaining term of Eq. (20) corresponds to a dimer isotherm with adsorption energy $E=\varepsilon_{1}$ $+\varepsilon_{2}\left(c=\sqrt{c_{1} c_{2}}\right)$. There are only two configurations with this energy for pairs of sites $(1,2)$ or $(2,1)$. Contrary to Eq. (19), Eq. (20) depends on $l$ and the dimer isotherm sees the topography.

For $l=1$ the adsorption energy of a dimer is $E=\varepsilon_{1}+\varepsilon_{2}$ for all configuration and, consequently, Eq. (20) is exact. However for $l>1$ and $c_{1} \neq c_{2}$ this equation is approximate. Then, to determine the range of validity of Eq. (20), we compare the analytic isotherm with MC results. Figure 4(a) shows the dimer isotherm for patches of size $l=2, c_{1}=10^{2}$, and different values of $c_{2}$. As we can see, for $c_{2} \geq 10$ the analytic isotherms agree very well with the MC data. However, for smaller values of $c_{2}$, the differences between theoretical and numerical data begin to be significant. This hap- 

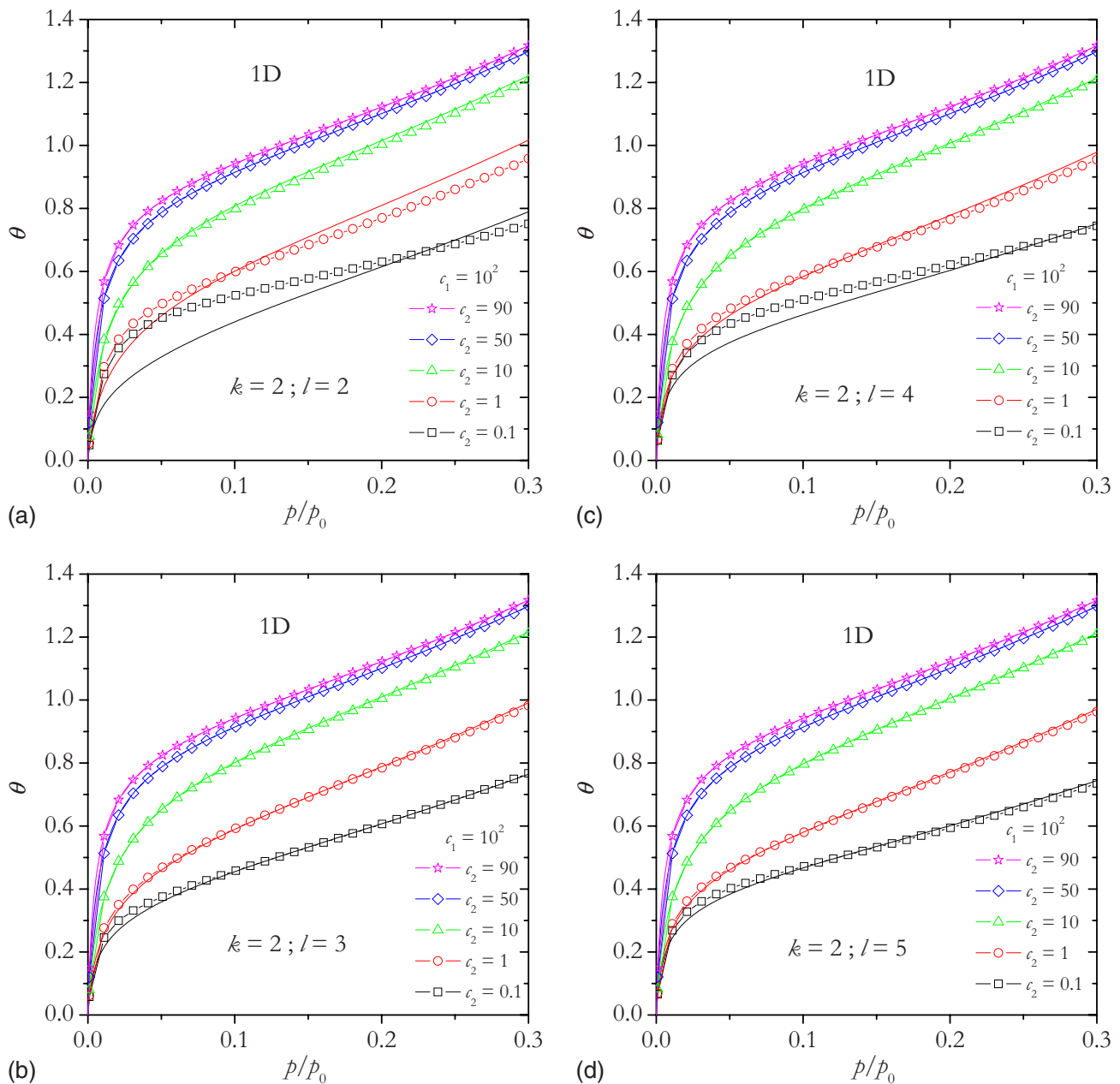

FIG. 4. (Color online) Comparison between approximate analytic and MC isotherms for dimers and different $1 \mathrm{D}$ heterogeneous surfaces: (a) $l=2$, (b) $l=3$, (c) $l=4$, and (d) $l=5$. In all cases we have used lattices of size $L=1200$ and $t=10^{5}$ MCSs.

pens because Eq. (20) has been built assuming that the three different pairs of sites are filled simultaneously and independently. However, for $c_{1} \gg c_{2}$, the real process occurs in three stages: (i) the pairs of sites $(1,1)$ are covered, (ii) the pairs $(2,2)$ begin to be filled, and (iii) the multilayer is formed. Note that in the first stage all the pair of sites $(1,2)$ and $(2,1)$ are removed. For this regime, a better approximation can be obtained by a semisum of two isotherms with $c_{1}$ and $c_{2}$.

When $l=3$ [Fig. 4(b)], the agreement between the analytic isotherms and the MC data is very good for all values of $c_{2}$. In this case, for $c_{1} \gg c_{2}$ the first stage does not eliminate all the pairs of sites $(1,2)$ and $(2,1)$, because each dimer occupies only two sites in the strong patches. For this reason the range of validity of Eq. (20) is wider than in the previous case. Now, for $l=4$ or $l=5$, we see in Figs. 4(c) and 4(d) that the behaviors are similar to those observed for $l=2$ or $l=3$, respectively. In general, for even $l$ the first stage eliminates almost completely the pairs of sites $(1,2)$ and $(2,1)$, while this does not happen for odd $l$. Finally, when $l \rightarrow \infty$ the fraction of pair $(1,2)$ and $(2,1)$ goes to zero and Eq. (20) is exact. This limit corresponds to the called large patches topography (LPT), where the surface is assumed to be a collection of homogeneous patches, large enough to neglect border effects between neighbor patches with different adsorption energies.
In general, if $k \gg l$ (with $k>1$ ), the multilayer adsorption isotherm can be represented by a single homogeneous isotherm,

$$
\theta=\theta_{\mathrm{loc}}\left(\sqrt{c_{1} c_{2}}\right) .
$$

On the other hand, for a LPT where $k \ll l$, the isotherm is

$$
\theta=\frac{1}{2} \theta_{\mathrm{loc}}\left(c_{1}\right)+\frac{1}{2} \theta_{\mathrm{loc}}\left(c_{2}\right) .
$$

The details of the topography are relevant only when $k \sim l$. In this case all terms in Eq. (16) are important. Nevertheless, it is also interesting to consider a simpler expression of the multilayer isotherm given by

$$
\theta=\left(\frac{l-1}{2 l}\right) \theta_{\mathrm{loc}}\left(c_{1}\right)+\left(\frac{1}{l}\right) \theta_{\mathrm{loc}}\left(\sqrt{c_{1} c_{2}}\right)+\left(\frac{l-1}{2 l}\right) \theta_{\mathrm{loc}}\left(c_{2}\right) .
$$

Equation (23) captures the extreme behaviors, Eqs. (22) and (21), and it approximates the MC isotherm as well as Eq. (16). To verify this statement, we calculate the integral 

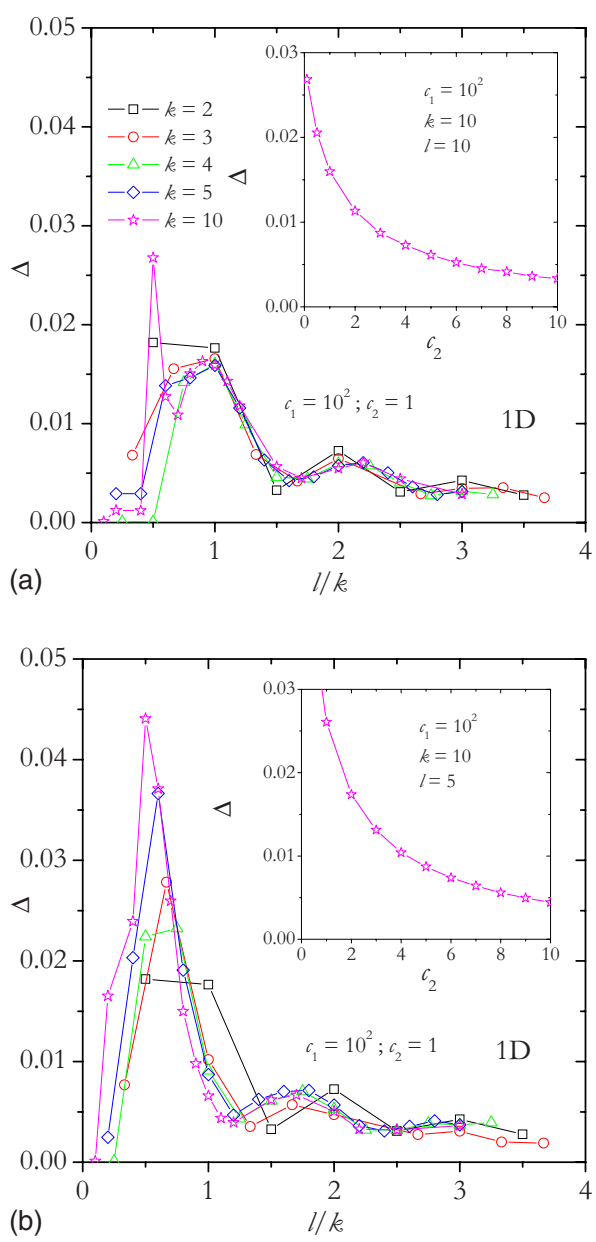

FIG. 5. (Color online) Function $\Delta$ vs $l / k$ for the adsorption in a 1D heterogeneous surface with $c_{1}=10^{2}, c_{2}=1$, and different values of $k$ as indicated (a) Function $\Delta$ calculated from Eq. (16). The inset shows the dependence of $\Delta$ on $c_{2}$ for $k=10$ and $l=10$. (b) Function $\Delta$ calculated from Eq. (23). The inset shows the dependence of $\Delta$ on $c_{2}$ for $k=10$ and $l=5$.

$$
\Delta=\int\left|\frac{\theta_{\mathrm{MC}}-\theta}{\theta_{\mathrm{MC}}}\right| d\left(p / p_{0}\right),
$$

which allows to quantify the difference between the MC isotherm, $\theta_{\mathrm{MC}}$, and the analytic isotherm, $\theta$, given by either the Eq. (16) or the new approach Eq. (23). For practical purposes, we have chosen a range of relative pressure of $0-0.3$ to calculate the integral Eq. (24). MC simulation were carried out for lattice sizes of $L=20 \mathrm{k}$ with a number of $t=10^{5}$ MCS.

Figure 5(a) shows the function $\Delta$ calculated from Eq. (16) for $c_{1}=10^{2}, c_{2}=1$, and different values of $k$ and $l$. As in the case of dimers, the difference between the analytic and the MC isotherms increases when $l$ is approximately a multiple of $k$. However, for large patches, i.e., $l>3 k$, this difference becomes smaller. The inset shows, for a particular case $(k=10$ and $l=10)$, how $\Delta$ diminishes as $c_{2}$ is increased. On the other hand, in Fig. 5(b) we can see the function $\Delta$ calculated from Eq. (23). For $k>2$, the first peak is higher than the one shown in Fig. 5(a) and is located in a value of $l / k$ between 0.5 and 0.7. Nevertheless, the oscillations attenuate quickly as the parameter $l / k$ is increased. As before, but now for $k=10$ and $l=5$, the inset shows how $\Delta$ diminishes as $c_{2}$

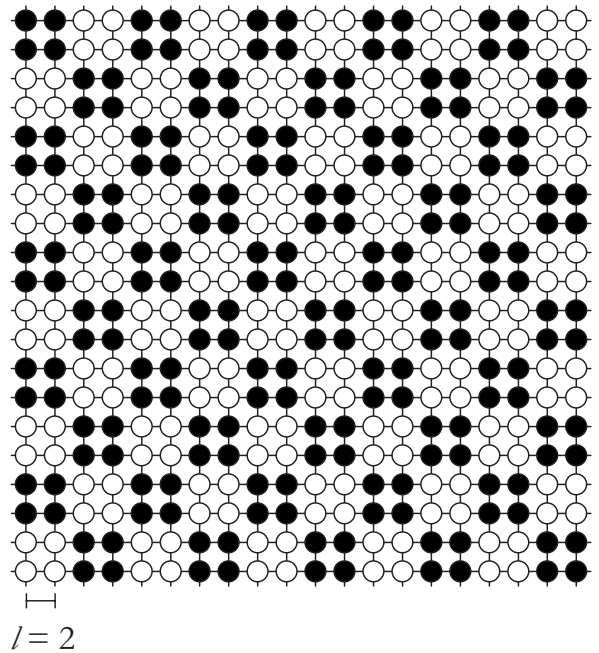

(a)

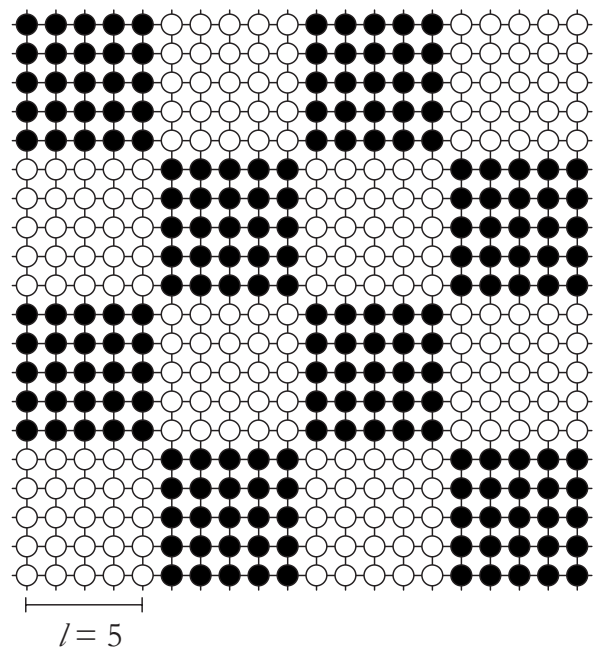

(b)

FIG. 6. Schematic of a bivariate surface with a chessboard topography: (a) $l=2$ and (b) $l=5$.

increases. Finally, the analysis of Fig. 5 indicates that, instead of Eq. (16), which has many terms, Eq. (23) can be used as a more simple expression to approach the 1D multilayer adsorption isotherm.

\section{B. Adsorption on 2D heterogeneous surfaces}

As in the homogeneous case, we represent the 2D surface by a square lattice with fully periodic boundary conditions. Strong and weak sites are spatially distributed in square patches of size $l$ forming a chessboard (see Fig. 6). Now, the total number of configurations available to a single $k$-mer on an empty lattice is $2 M$. However, as before, only $2 l$ terms are necessary to describe the multilayer isotherm. The explanation is quite simple: the available energies of a $k$-mer that it is forced to move in any direction of the lattice (row or column) are the same that in 1D. Then, Eq. (16) continues being valid in 2D, where the local isotherm is given by Eqs. (12) and (8), with $\gamma=4$.

We begin analyzing the multilayer isotherm for $k=2$ [for $k=1$, Eq. (19) continues being valid in 2D]. Unfortunately, it is not possible to write a simple analytic expression [as 


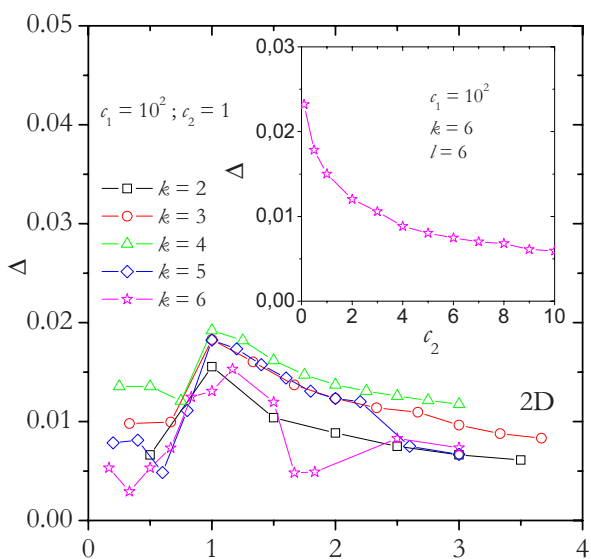

(a)

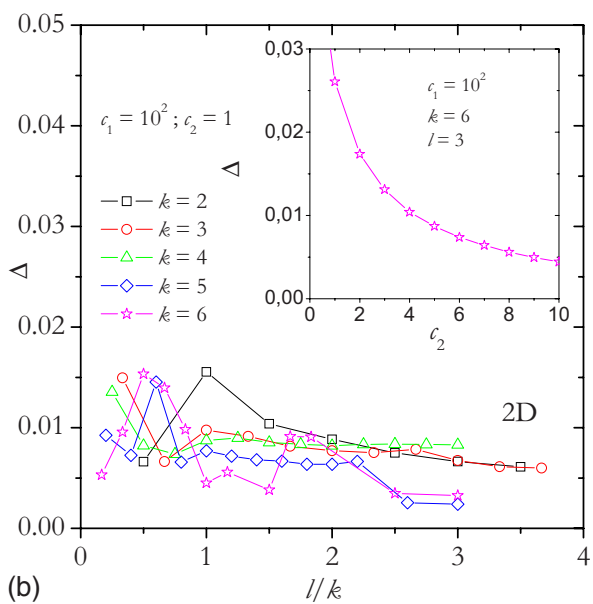

FIG. 7. (Color online) Function $\Delta$ vs $l / k$ for the adsorption in a $2 \mathrm{D}$ heterogeneous surface with $c_{1}=10^{2}, c_{2}=1$, and different values of $k$ as indicated. (a) Function $\Delta$ calculated from Eq. (16). The inset shows the dependence of $\Delta$ with $c_{2}$ for $k=6$ and $l=6$. (b) Function $\Delta$ calculated with Eq. (23). The inset shows the dependence of $\Delta$ with $c_{2}$ for $k=6$ and $l=3$.

Eq. (20)] in 2D. Nevertheless, the multilayer adsorption isotherm for dimers has the same structure as that of Eq. (20). Namely, it is composed by three terms with $c_{1}, c_{2}$ (both multiplied by $(l-1) / 2 l$ ), and $c=\sqrt{c_{1} c_{2}}$ (multiplied by $\left.1 / l\right)$. Note that in $2 \mathrm{D}$ this function is approximate for any value of the parameter $l$.

We compare the 2D dimer analytic isotherm with MC data, for $c_{1}=10^{2}$ and different values of $l$ and $c_{2}$. The behavior of these curves is very similar to the one observed in $1 \mathrm{D}$, but the difference between analytic and MC adsorption isotherms (for even and odd values of $l$ ) is no longer so important. On the other hand, Figs. 7(a) and 7(b) show the dependence of the function $\Delta$ on $l / k$, where $\Delta$ was calculated by using Eqs. (16) and (23), respectively. As in the case of 2D homogeneous surfaces, the analytic isotherm does not fit very well the MC data for $k>6$. For this reason, Figs. 7(a) and 7(b) show the function $\Delta$ up to $k=6$ only.

Summarizing, we have shown that just by using an expression of three terms, Eq. (23), we can approach very well the multilayer isotherm in 1D and 2D for the adsorption on heterogeneous surfaces. In the Sec. V, we will use this approximation and $\mathrm{MC}$ simulations to study how the topography affects the determination of monolayer volume predicted by the BET equation.

\section{MONOLAYER VOLUME}

In this section, we carry out numerical experiments to determine, in different adsorption situations, how much the value of the monolayer volume predicted by the BET equation differs from its real value, $v_{m}$. With this purpose, analytic and $\mathrm{MC}$ isotherms were analyzed as experimental data. In this way, we determined how adsorbate size, energetic heterogeneity, and surface topography affect the standard determination of the monolayer volume.

In a typical experiment of adsorption, the adsorbed volume of the gas, $v$, is measured at different pressures and at a given fixed temperature. In terms of this quantity, the total coverage is $\theta=v / v_{m}$. Analyzing an isotherm with the BET equation, it is possible to estimate the monolayer volume. We rewrite Eq. (4) as

$$
\frac{p / p_{0}}{v\left(1-p / p_{0}\right)}=\frac{1}{c v_{m}}+\frac{(c-1)}{c v_{m}} p / p_{0} .
$$

This equation is a linear function of $p / p_{0}$. If we denote with $a$ and $b$, the $y$-intercept and the slope of this straight line, respectively, we obtain

$$
v_{m}^{*}=\frac{1}{a+b}
$$

and

$$
c^{*}=\frac{b}{a}+1
$$

The asterisk has been added in order to indicate that the quantities given by Eqs. (26) and (27) correspond to the prediction of the BET theory. Then, by means of a plot (the so-called BET plot) of the experimental data of $p / p_{0} / v(1$ $\left.-p / p_{0}\right)$ versus $p / p_{0}$, we can obtain an estimate of the monolayer volume and the parameter $c$. Nevertheless, in the experiments it is commonly found that there are deviations from linearity in the BET plot. In many cases, the linear range extends from a relative pressure of $0.05-0.35$, although there are cases where the range is shorter. ${ }^{1}$

Although the BET plot is a very simple and popular protocol, the value of the monolayer volume obtained in this way can differ from its real value. ${ }^{13-15}$ In the following, we will show that even for adsorption over homogeneous surfaces, the polyatomic character of the adsorbate affects significantly the predictions of a BET plot. Next, in Sec. V B, by considering bivariate surfaces, we will study the combined effect of energetic heterogeneity and multisite occupancy.

\section{A. Homogeneous surfaces}

We begin analyzing the BET plots of the multilayer adsorption of $k$-mers over homogeneous surfaces, given by Eqs. (8) and (12) and MC data. Although in each particular case it is possible to find an optimum range of relative pressures, we have chosen, for practical purposes, to set this range from 0.05 to 0.25 . Nevertheless, by choosing other 


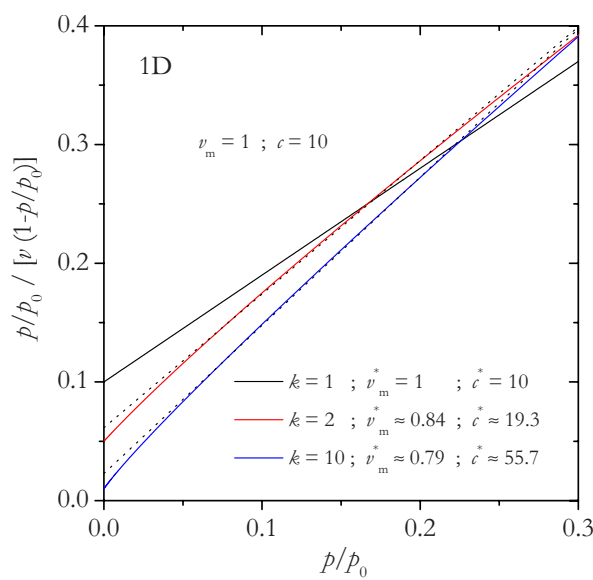

FIG. 8. (Color online) BET plots for 1D analytic isotherms of $k$-mers with $v_{m}=1, c=10$, and different values of $k$ as indicated. Dotted lines correspond to linear fits of the data in the range $0.05-0.25$.

ranges (for example, between 0.05 and 0.35 ) we obtain similar results.

In Fig. 8 we show the BET plot for 1D analytic isotherms with $c=10$ and $k=1,2$, and 10 . Note the deviations from linearity in the isotherms for $k=2$ and 10 , which are concave to the pressure axis. The same behavior is observed in experimental isotherms and it is attributed to the existence of surface heterogeneities. ${ }^{13}$ However, as we see in the ex-
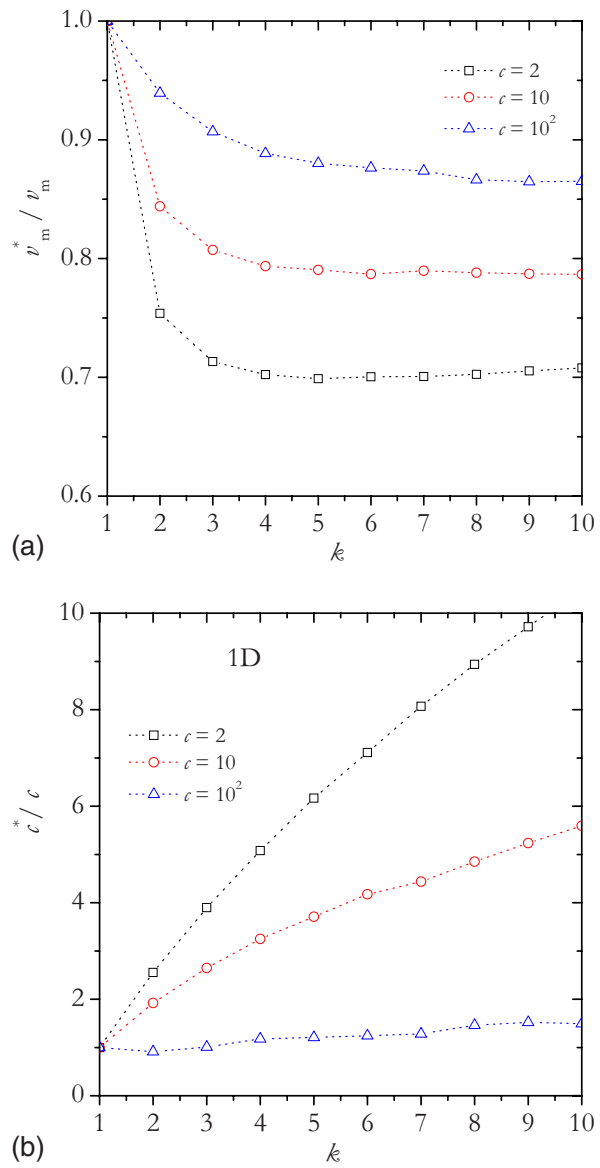

FIG. 9. (Color online) Results of the BET plots for the adsorption in 1D homogeneous surfaces. Dependence on $k$ of the fractions, (a) $v_{m}^{*} / v_{m}$ and (b) $c^{*} / c$, for three different values of $c$ as indicated.
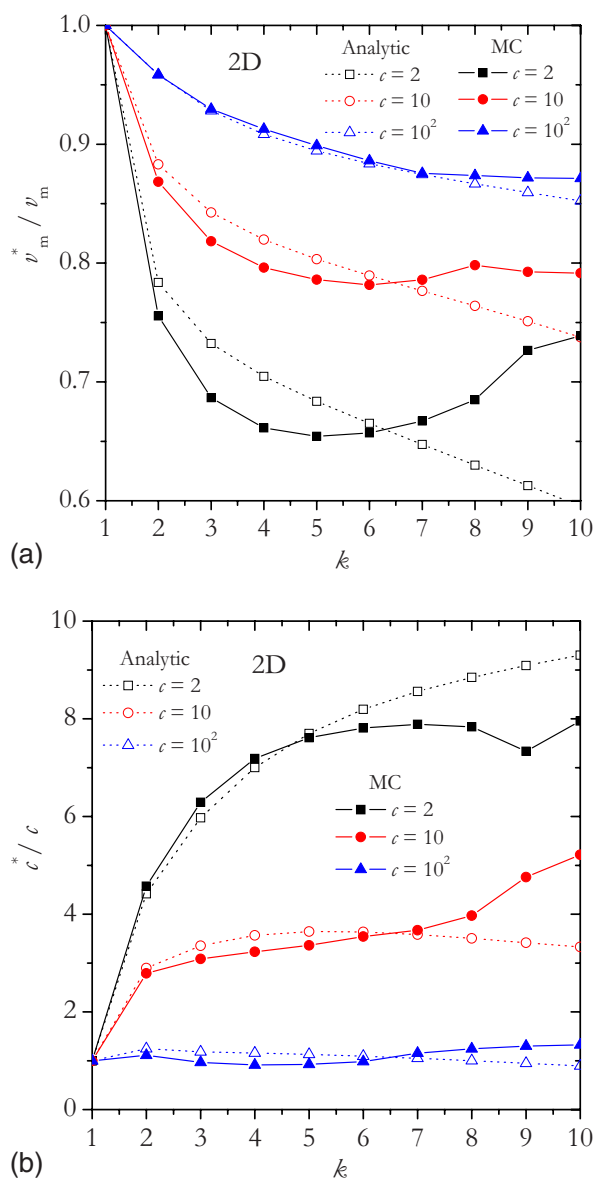

FIG. 10. (Color online) Results of the BET plots for the adsorption in 2D homogeneous surfaces. Dependence on $k$ of the fractions, (a) $v_{m}^{*} / v_{m}$ and (b) $c^{*} / c$, for three different values of $c$ as indicated.

ample shown in Fig. 7, these deviations also appear for the multilayer adsorption with multisite occupancy on a homogeneous surface.

On the other hand, as indicated in Fig. 8, the obtained value of $v_{m}^{*}$ for $k>1$ is smaller than the real one (we set $v_{m}=1$ ), while the opposite effect is observed in the estimate of the parameter $c$. Figures 9(a) and 9(b) show the dependence of these quantities on $k$ for different values of $c$. In all cases, we obtain $v_{m}^{*} \leq v_{m}$ and $c^{*} \geq c$, but the differences between the BET predictions and the real values are smaller with increasing $c$.

Similar results have been obtained in 2D: the BET plots of both analytic and MC isotherms show the same curvature as found in 1D. Figures 10(a) and 10(b) show the results of these 2D BET plots. As we can see, the differences between analytic and MC isotherms are significant for small values of $c$. However, always $v_{m}^{*} \leq v_{m}$ and $c^{*} \geq c$ for $k>1$. As in the $1 \mathrm{D}$ case, the monolayer volume predicted by BET is approximately $10 \%-30 \%$ smaller than the real value.

\section{B. Heterogeneous surfaces}

In previous work ${ }^{13-15}$ it has been determined that, as heterogeneous adsorption isotherms are analyzed, the monolayer volume obtained from a BET plot is smaller than the real value. In all theses cases, always random heterogeneous surfaces were considered and only the multisite occupancy of 

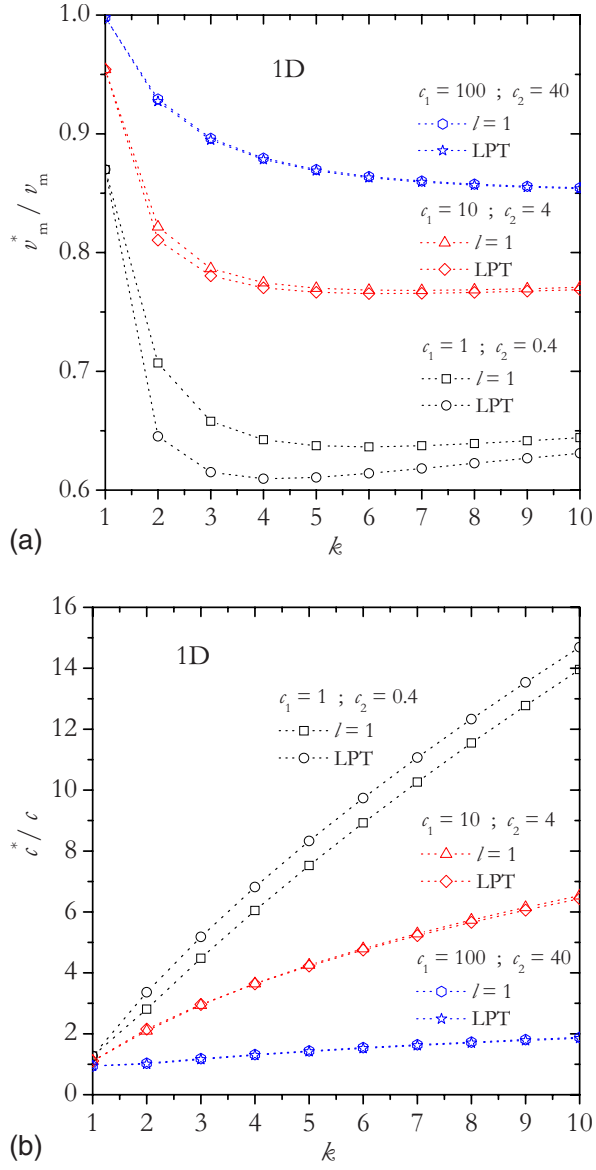

FIG. 11. (Color online) Results of the BET plots for the adsorption in 1D heterogeneous surfaces with $l=1$ and LPT. Dependence on $k$ of the fractions, (a) $v_{m}^{*} / v_{m}$ and (b) $c^{*} / c$, for three different values of $c_{1}$ and $c_{2}$ (being $\left.c_{1} / c_{2}=2.5\right)$ as indicated.

the adsorbate was taken into account in Ref. 15. In this section, we study the dependence of the monolayer volume on both adsorbate size and surface topography. In particular, we analyze analytic and MC adsorption isotherms of $k$-mers over 1D and 2D bivariate patchwise surfaces with $l=1$ and LPT (the MC simulation for a LPT were carried out on big lattices with only two patches).

Figures 11(a) and 11(b) show the results of the 1D BET plots for three different values of $c_{1}$ and $c_{2}$, being $c_{1} / c_{2}$ $=2.5$. In this case, only analytic isotherms were studied because they are exact for LPT and the agreement with MC data is seen to be remarkably good for $l=1$ [except for odd values of $l(l>1)$, as was previously mentioned]. In all cases we used $c=\sqrt{c_{1} c_{2}}$ as the reference parameter. As we can see, the curves show that there is not a significant difference between both topographies. Only when the quotient between $c_{1}$ and $c_{2}$ is increased, the space distribution of the adsorption energies over the solid surface begins to be important. This is shown in Figs. 12(a) and 12(b), where $c_{1} / c_{2}=10^{3}$. The results of the BET plots for $l=1$ and LPT are very different. For $c_{1}=10$ and $c_{2}=0.01$, the deviations due to molecule size $k$ are increased in LPT, i.e., the monolayer volume and the parameter $c$ obtained from a BET plot are, respectively, smaller and larger than the real values (or the reference value). However, most of the curves show a compensation effect which is larger for $l=1, c_{1}=10^{3}$, and $c_{2}=1$.
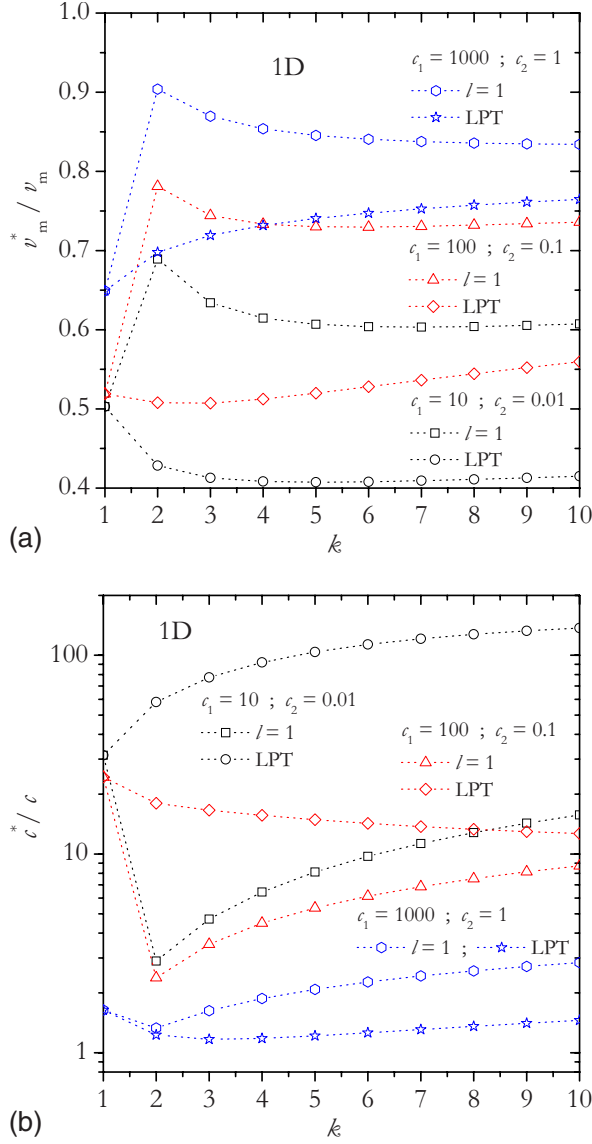

FIG. 12. (Color online) Results of the BET plots for the adsorption in 1D heterogeneous surfaces with $l=1$ and LPT. Dependence on $k$ of the fractions, (a) $v_{m}^{*} / v_{m}$ and (b) $c^{*} / c$, for three different values of $c_{1}$ and $c_{2}$ (being $\left.c_{1} / c_{2}=10^{3}\right)$ as indicated.

Finally, in 2D we studied both analytic and MC isotherms for the same parameters analyzed in 1D. We obtained a similar behavior to the $1 \mathrm{D}$ case; even taking very different values of the parameters $c_{1}$ and $c_{2}$, and considering the two extreme topographies ( $l=1$ and LPT), it is not possible to obtain a complete compensation effect.

\section{ADSORPTION ON CARBON NANOTUBE BUNDLES}

Now, we shortly consider the adsorption on carbon nanotube bundles. In these systems, the isosteric heat of adsorption is generally a decreasing function of coverage, showing that adsorption energies are larger than lateral interactions. ${ }^{29}$ Also, nanotubes provide experimental realization of 1D substrates, because these systems can exhibit large aspect ratios and nanoscale cross section. ${ }^{30}$ In this context, a lattice-gas theory of multilayer adsorption of noninteracting particles on heterogeneous surfaces as the one presented in this work, it could be appropriate to study the adsorption on carbon nanotube bundles.

Figure 13 shows the 1D dimer isotherm Eq. (20) for a LPT and four temperatures between 80 and $200 \mathrm{~K}$. A value of $\varepsilon_{1}=150 \mathrm{meV}$ has been chosen to mimic the adsorption of ethane on samples of single-walled carbon nanotubes, where the more negative binding energy was found to be 308 $\mathrm{meV} .{ }^{29}$ We supposed that the adsorption energy on weak sites 


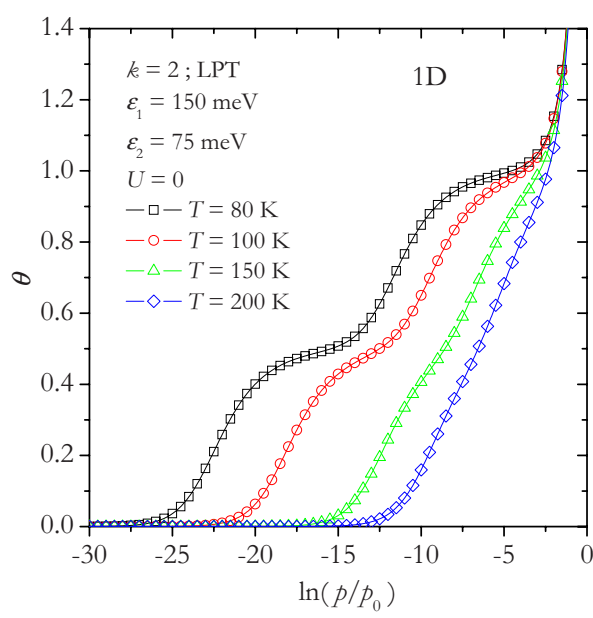

FIG. 13. (Color online) 1D dimer (analytic) isotherms, on a semilogarithmic scale, for a LPT with adsorption energies $\varepsilon_{1}=150 \mathrm{meV}, \varepsilon_{2}=75 \mathrm{meV}$, and $U=0$. Figure shows the isotherms for four temperatures as indicated.

is $50 \%$ smaller $^{31}$ than on strong sites, $\varepsilon_{2}=75 \mathrm{meV}$, and that $U=0$ (notice that the $\varepsilon_{i}$ are the adsorption energies per site, then for $k=2,2 \varepsilon_{1}=300 \mathrm{meV}$, and $2 \varepsilon_{2}=150 \mathrm{meV}$ ). On a semilogarithmic scale, it is possible to observe the presence of two rounded steps at $80 \mathrm{~K}$, which disappear when temperature is increased. Roughly, a similar behavior is observed in experimental isotherms. ${ }^{29}$ Although the $1 \mathrm{D}$ model is not the most appropriate to describe the adsorption in nanotube bundles, Fig. 13 suggests that a more elaborate model (with the incorporation of 2D terms representing the adsorption on the cylindrical outer surface of individual nanotubes, for example) could be useful to study these systems.

\section{CONCLUSIONS}

In the present paper, we studied a model of multilayer adsorption on 1D and 2D homogeneous and heterogeneous patchwise bivariate surfaces of noninteracting polyatomic species. Following a simple formalism analytic isotherms were calculated. These formalism reproduces the classical BET theory ${ }^{6}$ and the recently reported dimer equations ${ }^{17}$ leads to the exact solution for a 1D homogeneous substrate and, as is demonstrated from comparison with MC simulation, provides a good approximation for 1D heterogeneous surfaces. With respect to $2 \mathrm{D}$ substrates (homogeneous and heterogeneous surfaces), the approach is not exact. However, MC data shows that for molecules of moderate size (not larger than $k=6$ ), the analytic isotherm behaves qualitatively similar to the simulation.

In addition, we carry out numerical experiments to determine, in different adsorption situations, how much the value of the monolayer volume predicted by the BET equation differs from its real value. For this purpose, analytic isotherms and MC data were analyzed as experimental data. For 1D and 2D homogeneous surfaces, the monolayer volume calculated by the BET plots is approximately $10 \%-30 \%$ smaller than the real value. On the other hand, in all cases, the parameter $c^{*}$ is always larger than $c$. As the multilayer adsorption occurs on a bivariate patchwise heterogeneous surface, a compensation effect is found. Nevertheless, in any of the considered cases, this compensation is not enough to eliminate the decrease caused by the molecular size.

Finally, we analyze the 1D model for binding energy and temperature characteristics of the adsorption of ethane on samples of single-walled nanotubes. The isotherms show two rounded steps, which disappear when temperature is increased. A similar behavior was observed in experiments, stimulating the development of more elaborate lattice-gas models (including, for example, 2D terms representing the adsorption on the cylindrical outer surface of individual nanotubes), which can be able to reproduce real experimental conditions.

\section{ACKNOWLEDGMENTS}

This work was supported in part by CONICET (Argentina) under Project No. PIP 6294, Universidad Nacional de San Luis (Argentina) under Project No. 322000, Universidad Tecnológica Nacional, Facultad Regional San Rafael (Argentina) under Project No. PID PQCO SR 563 and the National Agency of Scientific and Technological Promotion (Argentina) under Project No. 33328 PICT 2005.

${ }^{1}$ S. J. Gregg and K. S. W. Sing, Adsorption, Surface Area and Porosity (Academic, New York, 1991).

${ }^{2}$ W. Rudziński and D. H. Everett, Adsorption of Gases on Heterogeneous Surfaces (Academic, London, 1992).

${ }^{3}$ J. Frenkel, Kinetic Theory of Liquids (Clarendon, Oxford, 1946); J. Frenkel, Kinetic Theory of Liquids (Dover, New York, 1955).

${ }^{4}$ G. D. J. Halsey, Chem. Phys. 16, 931 (1948).

${ }^{5}$ T. L. Hill, Adv. Catal. 4, 211 (1952).

${ }^{6}$ S. Brunauer, P. H. Emmett, and E. J. Teller, J. Am. Chem. Soc. 60, 309 (1938).

${ }^{7}$ T. L. Hill, An Introduction to Statistical Thermodynamics (Addison Wesley, Reading, 1962).

${ }^{8}$ W. A. Steele, The Interaction of Gases with Solid Surfaces (Pergamon, New York, 1974).

${ }^{9}$ A. W. Adamson, Physical Chemistry of Surfaces (Wiley, New York, 1990).

${ }^{10}$ J. B. Parra, J. C. de Sousa, R. C. Bansal, J. J. Pis, and J. A. Pajares, Adsorpt. Sci. Technol. 51, 12 (1995).

${ }^{11}$ P. J. Pomonis, D. E. Petrakis, A. K. Ladavos, K. M. Kolonia, G. S. Armatas, S. D. Sklari, P. C. Dragani, A. Zarlaha, V. N. Stathopoulos, and A. T. Sdoukos, Microporous Mesoporous Mater. 69, 97 (2004).

${ }^{12}$ J. Rouquerol, P. Llewellyn, and F. Rouquerol, Stud. Surf. Sci. Catal. 160, 49 (2007).

${ }^{13}$ W. C. Walker and A. C. Zettlemoyer, J. Phys. Colloid Chem. 52, 47 (1948).

${ }^{14}$ J. Cortés and P. Araya, J. Colloid Interface Sci. 115, 271 (1987).

${ }^{15}$ P. Nikitas, J. Phys. Chem. 100, 15247 (1996).

${ }^{16}$ G. D. García, F. O. Sánchez-Varretti, F. Romá, and A. J. Ramirez-Pastor, Surf. Sci. 603, 980 (2009).

${ }^{17}$ J. L. Riccardo, A. J. Ramirez-Pastor, and F. Romá, Langmuir 18, 2130 (2002).

${ }^{18}$ F. Romá, A. J. Ramirez-Pastor, and J. L. Riccardo, Surf. Sci. 583, 213 (2005).

${ }^{19}$ L. E. Firment and G. A. Somorjai, J. Chem. Phys. 66, 2901 (1977).

${ }^{20}$ L. E. Firment and G. A. Somorjai, J. Chem. Phys. 69, 3940 (1978).

${ }^{21}$ G. A. Somorjai and M. A. Van Hove, Adsorbed Monolayers on Solid Surfaces (Springer-Verlag, Berlin, 1979).

${ }^{22}$ Z. Wu, S. N. Ehrlich, B. Matthies, K. W. Herwing, P. Dai, U. G. Volkmann, F. Y. Hansen, and H. Taub, Chem. Phys. Lett. 348, 168 (2001).

${ }^{23}$ A. J. Phares and F. J. Wunderlich, Phys. Lett. A 121, 347 (1987).

${ }^{24}$ A. J. Ramirez-Pastor, T. P. Eggarter, V. D. Pereyra, and J. L. Riccardo, Phys. Rev. B 59, 11027 (1999).

${ }^{25}$ F. Romá, J. L. Riccardo, and A. J. Ramirez-Pastor, Langmuir 22, 3192 (2006).

${ }^{26}$ J. L. Riccardo, F. Romá, and A. J. Ramirez-Pastor, Int. J. Mod. Phys. B 
20, 4709 (2006).

${ }^{27}$ J. L. Riccardo, M. A. Chade, V. D. Pereyra, and G. Zgrablich, Langmuir 8, 1518 (1992).

${ }^{28}$ F. Bulnes, A. J. Ramirez-Pastor, and G. Zgrablich, J. Chem. Phys. 115, $1513(2001)$
${ }^{29}$ D. S. Rawat and A. D. Migone, Phys. Rev. B 75, 195440 (2007).

${ }^{30}$ S. M. Gatica, M. M. Calbi, R. D. Diehl, and M. W. Cole, J. Low Temp. Phys. 152, 89 (2008)

${ }^{31}$ D. S. Rawat, M. M. Calbi, and A. D. Migone, J. Phys. Chem. C 111, 12980 (2007) 